\title{
Una aproximación teórica a la innovación. Fuentes y barreras en el sector lácteo*
}

\section{A theoretical approach to innovation. Sources and barriers in the dairy sector}

\author{
Carlos Augusto Rincón Díaz** \\ Rubén Darío Díaz Mateus***
}

Recibido: 15 de febrero de 2016

Revisado: 16 de marzo de 2016

Aprobado: 28 de abril de 2016

* Este artículo es resultado del proyecto de investigación: Innovación y energía en las unidades productivas del sector lácteo, financiado por la Facultad de Ciencias Administrativas y Contables de la Universidad de La Salle. Cómo citar este artículo: Rincón Díaz, C. A. \& Díaz Mateus, R. D. (2016). Una aproximación teórica a la innovación.Fuentes y barreras en el sector lácteo. Revista CIFE, 18(28), 91-110. (DOI: http://dx.doi.org/10.15332/ s0124-3551.2016.0028.04

** Docente investigador del Programa de Administración de Empresas de la Facultad de Ciencias Administrativas y Contables de la Universidad de La Salle. Economista de la Universidad Piloto. Doctor en Gestión de Empresas de la Universidad Politécnica de Valencia (España). Correo electrónico: carincon@unisalle.edu.co

*** Docente investigador del Programa de Contaduría Pública y líder del grupo de investigación Desarrollo y Sociedad de la Facultad de Ciencias Administrativas y Contables de la Universidad de La Salle. Economista de la UPTC. Magíster en Fundamentos de Economía de la Universidad Santiago de Compostela (España). Estudiante del doctorado en Agrociencias de la Universidad de La Salle (Colombia).Correo electrónico: rudiaz@unisalle.edu.co 


\section{Resumen}

Las distintas problemáticas del sector agropecuario en Colombia requieren de la configuración de conglomerados productivos menos vulnerables y con el acompañamiento de una formulación de políticas públicas que disminuyan la brecha entre la investigación y la adopción de tecnología. A pesar de que el gobierno está trabajando en mejorar la Política de Ciencia, Tecnología e Innovación para el sector agropecuario, orientándola a atender la baja productividad, uno de los sectores que aún enfrenta de forma acentuada estas discrepancias es el sector lácteo. En dicho contexto, este artículo tiene por objetivo presentar un esquema teórico de la innovación como referente para la toma de decisiones de los diferentes agentes participantes en la cadena láctea. Para tal propósito, se ha realizado una revisión de la literatura sobre innovación y en ella se han identificado las principales fuentes y barreras para analizarlas en el contexto de la cadena láctea. En conclusión, en la cadena láctea existe una relación entre las fuentes y las barreras identificadas en la literatura de la innovación.

Palabras clave: Innovación, cadena de valor, sector lácteo.

Glasificación JEL: Q13, Q18, R14

\section{Abstract}

The different problems of the agricultural sector in Colombia require the formation of less vulnerable productive conglomerates and with the assistance of a formulation of public policies that reduce the gap between research and adoption of technology. Despite the fact that the government is working to improve the Science, Technology and Innovation Policy for the agricultural sector, in order to address low productivity, one of the sectors that still faces these differences is the dairy sector. In this context, this article aims to present a theoretical framework of innovation as a reference for the decisionmaking of the different actors involved in the dairy chain. For this purpose, a review of the literature on innovation has been carried out and it has identified the main sources and barriers to analyze them in the context of the dairy chain. In conclusion, in the dairy chain there is a relationship between the sources and the barriers identified in the innovation literature.

Keywords: Innovation, value chain, dairy sector.

Classification JEL: Q13, Q18, R14 


\section{Introducción}

El reto de crecimiento en términos biológicos y económicos, desde la perspectiva de las otras especies vivas frente al comportamiento del ser humano, no permite actualmente establecer relaciones de equilibrio, sin embargo, el ser humano modifica su realidad para mantener estados de bienestar y supervivencia. La ley natural de la biósfera impone límites en el uso de recursos naturales, por tanto, hace necesario conocer con precisión no solo el comportamiento de los ecosistemas, sino las relaciones del ser humano con sus organizaciones socioeconómicas; así, estudiar las problemáticas ambientales desde una sola disciplina no permite ir más allá de los escenarios de contaminación. Según Azqueta (2002), los problemas ambientales tienen una multitud de fuentes generadoras; sin embargo, los modos de consumo y producción del sistema económico de una sociedad aceleran otra clase de problemas ambientales a nivel global, regional y local donde están expuestas tanto las sociedades desarrolladas como las que están en vía en desarrollo.

La Política de Ciencia, Tecnología e Innovación para el sector agropecuario en Colombia está orientada a atender la baja productividad del sector. Los escenarios de la configuración en conglomerados productivos son vulnerables ante las problemáticas socioeconómicas tanto internas como externas propias de la cadenada láctea, lo cual incentiva la formulación de una política pública que permita disminuir la brecha entre la investigación y la adopción de conocimiento y tecnología para mejorar la productividad de esta. La investigación dentro de la cual se enmarca este artículo tiene como propósito identificar las fuentes y barreras asociadas con la innovación en las unidades productivas de los diferentes eslabones de la cadena láctea.

Los desarrollos de innovación en el sector lácteo, en especial en el eslabón primario, son incipientes o no son identificados como actividades propias relacionadas con la innovación. No obstante, el eslabón secundario y el terciario tienen mayor capacidad para invertir en investigación y contribuyen a la creación de procesos innovadores que no se transfieren al primer eslabón. En este contexto, este artículo presenta un esquema teórico de la innovación como referente para la toma de decisiones de los diferentes agentes participantes en la cadena láctea.

Este documento se estructura en tres partes. En primer lugar, se presenta la fundamentación teórica relacionada con la innovación y la perspectiva de la cadena láctea; posteriormente se describe la metodología empleada y se realiza una discusión relacionada con las fuentes y barreras de la innovación en la cadena láctea; finalmente, se muestran algunas conclusiones. 


\section{Antecedentes del problema}

Según el ranking de competitividad del Foro Económico Mundial 2015, Colombia ocupa el puesto 61 y está por debajo de países de la región como Chile y México (Foro Económico Mundial, 2016). La baja competitividad del país es resultado del atraso en la infraestructura, carencias en la educación, baja capacidad innovadora y nivel tecnológico, además de la alta informalidad del tejido empresarial conformado, en su mayoría, por pequeñas y medianas empresas (alrededor del $93 \%$ ). Estas condiciones determinan la capacidad que tienen nuestras empresas para adaptarse a las cambiantes condiciones de los mercados, en especial aquellas que se encuentran en entornos rurales o cuya actividad se centra en la producción, trasformación y comercialización de productos primarios.

Con el objeto de contribuir a mejorar esta situación, el Plan Nacional de Desarrollo 20142018 (DNP, 2011 ), dentro de un esquema de construcción de la paz para Colombia, en su capítulo VI titulado "Crecimiento verde", propone un esquema estratégico enfocado en la transformación del campo, entendiendo que el crecimiento económico que el país requiere depende del desarrollo de nuestro potencial agrario. Para alcanzar esta meta, es necesaria la participación de distintos agentes, como son el Gobierno, el sector financiero, el sector externo, las empresas, el sector educativo y centros de investigación.

Según el Programa de las Naciones Unidas para el Desarrollo (PNUD, 2011), la estructura agraria de Colombia se caracteriza por el conflicto en la ocupación económica y social del territorio y por presentar comportamientos ineficientes que se representan en baja productividad, uso inadecuado de los recursos naturales, alta concentración de la propiedad, niveles de pobreza y exclusión superiores a los observados en las zonas urbanas. Así, las diferencias en los estilos de vida entre las grandes y las pequeñas urbes tienden a ampliar las brechas socioeconómicas y ambientales y dan como resultado alteraciones en los territorios, las cuales se evidencian en nuevos encadenamientos urbanos rurales, empleo rural no agrícola, provisión de servicios ambientales, construcción de cadenas agroambientales, pueblos como centros de servicios, roles comunitarios y sociales exclusivos de la actividad agrícola y uso inadecuado de fuentes energéticas requeridas en estas nuevas dinámicas Rosas-Baños (2013).

Como consecuencia de lo vulnerables que son las unidades productoras rurales y de la fragilidad del sector ante los cambios del mercado, de políticas públicas y del cambio climático, se deben visualizar perspectivas del desarrollo rural incluyentes y sostenibles en escenarios de cadenas productivas. En el informe Colombia rural. Razones para la esperanza. Informe Nacional de Desarrollo Humano (PNUD, 2011), se describen las brechas urbano-rurales: el $80 \%$ de los propietarios-poseedores están prácticamente en la pobreza absoluta, pues obtienen un ingreso que no supera medio salario mínimo legal; en los centros urbanos, en promedio, la pobreza es 2,3 veces menor que en municipios 
de alta ruralidad; las tasas de cobertura bruta en educación media en los municipios de alta ruralidad son 2,7 veces menores que en los centros urbanos; en las zonas rurales de Colombia solamente se utiliza el 22,7 \% de la superficie apta para actividades agrícolas y silvoagrícolas; el $65 \%$ de los campesinos que están en situación de desplazamiento son menores de 25 años (PNUD, 2011, pp. 64-66).

La complejidad de los sistemas productivos rurales, en especial la cadena láctea, está asociada con la dinámica del mercado interno y la integración de los diferentes eslabones que permitan satisfacer la expansión de demanda y el consumo de productos lácteos. La tendencia más significativa hacia la producción y la transformación láctea se define en la organización del sistema productivo, cerca de los centros urbanos, para potenciar el apalancamiento en el consumo. No obstante, la creciente tecnificación del proceso productivo con miras a mejorar la productividad de los sistemas de producción implica la incorporación de nuevas tecnologías, tales como ordeños mecánicos, tanques de enfriamiento, entre otras, que requieren de energía y materia externa para su funcionamiento. En esa medida, se prevé que este rubro sea representativo en los costos de producción láctea y en la competitividad en los mercados internacionales; en Colombia esta práctica permea los procesos productivos como procesos innovadores al entrar en asocio con la tecnología y la maquinaria que vienen desde afuera, situación negativa por la no identificación del conocimiento de procesos internos generadores de valor en los diferentes eslabones de la cadena láctea.

Del mismo modo, el diagnóstico realizado y citado en los estudios del Departamento Nacional de Planeación (DNP) y Colciencias describe como problema central en los sistemas productivos rurales la baja capacidad en gestión del conocimiento en las organizaciones, principalmente en identificar, producir, difundir, usar e integrar conocimiento, innovación y tecnología aplicable al sector productivo, en especial su apropiación en el sector rural. Así mismo, las organizaciones rurales presentan limitantes de acceso a nuevas tecnologías, resistencia cultural de adaptación, perspectivas de alto riesgo al invertir en innovación y tecnología, y como resultado de estas conductas se evidencia una baja productividad, especialmente de los pequeños productores, de manera que se pierden oportunidades de integración para crear valor en la cadena láctea (DNP, 2009).

En consecuencia, la Política de Ciencia, Tecnología e Innovación para el sector agropecuario en Colombia está orientada a atender la baja productividad del sector, la cual se ha dado como resultado de las dinámicas del mercado internacional. En este escenario, las organizaciones integrantes de la cadena láctea adquieren el reto de la investigación e innovación tecnológica para articularse entre los diferentes eslabones con la finalidad de aumentar la competitividad en el mercado interno y en el externo. Las debilidades evidentes en los diagnósticos resaltan la influencia de los altos costos de producción, la baja productividad en relación con los principales actores internacionales, la dispersión en la producción primaria, el mínimo nivel de asociatividad en los eslabones de la cadena, la 
alta informalidad en la comercialización y transformación de la leche y sus derivados, el bajo nivel de diversificación de productos y destinos de exportación y un deficiente estatus sanitario en relación con las exigencias de los mercados; por ello se requiere la formulación de estrategias para consolidar la competitividad del subsector, tanto en el mercado interno como en el externo (DNP, 2010b, p. 4).

Por consiguiente, se hace necesario revisar la generación y la transferencia de conocimiento e innovación en los distintos eslabones de la cadena láctea, no solo los basados en los avances tecnológicos focalizados para sobreponer las barreras impuestas por el clima, la cultura, aspectos geográficos y sociales. Por esta razón, la intervención de los diferentes agentes desde una perspectiva de innovación que integre la relación entre las fuentes y las barreras de la innovación permitirá establecer las configuraciones de los ecosistemas innovadores rurales entre las diferentes cadenas productivas de la economía rural de Colombia. En otras palabras, desde el marco conceptual de la innovación surge el siguiente cuestionamiento: ¿cuáles son las fuentes y las barreras asociadas con la innovación en la cadena láctea?

\section{Fundamentación teórica}

\subsection{La cadena de valor}

Los territorios debían especializarse en actividades económicas que, en función de sus recursos, les representen menos costos y mayores ventajas en economías de escala (Krugman, et al. 2012). Bajo esta visión neoclásica, distintas investigaciones (Hirschman, 1958; Porter, 1985; Krugman, 1999; Kaplinsky y Morris, 2000) han expuesto que para entender la dinámica de explotación económica de los territorios se deben estudiar las interacciones entre distintos elementos o eslabones que son determinantes para la creación de valor. Para Porter (1985), el concepto de cadena de valor al estudiar los eslabones de una actividad económica que operan de forma desagregada y las estrategias que esta puede adoptar para alinear las actividades de manera que pueda maximizar su efecto en dichos eslabones con resultados maximizadores de beneficios. La cadena de valor de una organización forma parte de un sistema de valor más grande en el que se integran e interactúan las diferentes cadenas de valor de proveedores, canales de distribución, el comprador final y la organización, determinando en muchos casos la forma en que esta se puede gestionar de forma eficiente, tanto en lo económico como en lo social. En consecuencia, la obtención y el mantenimiento de la ventaja competitiva dependerán no solo de comprender la cadena de valor de una organización, sino cómo la organización encaja en el sistema de valor general de un territorio. 
Porter (1985) indicó que la cadena de valor está constituida por tres elementos: las actividades primarias, involucradas con el desarrollo del producto, la producción, la logística, la venta y la posventa; las actividades de soporte, que integran la administración de los recursos humanos, compras de bienes y servicios, desarrollo tecnológico, finanzas, contabilidad, relaciones públicas, asesoría legal y la gerencia general; y finalmente está el margen o la diferencia que se obtienen entre el valor total y los costos totales. La figura 1 muestra la interacción entre estos elementos.

Figura 1. Esquema de la cadena de valor

\begin{tabular}{|c|c|c|c|c|c|}
\hline & \multicolumn{5}{|c|}{ Infraestructura de la empresa } \\
\hline \multirow{5}{*}{$\begin{array}{l}\text { Actividades } \\
\text { de apoyo }\end{array}$} & \multicolumn{5}{|c|}{ Gestión de recursos humanos } \\
\hline & \multicolumn{5}{|c|}{ Servicios generales } \\
\hline & \multicolumn{5}{|c|}{ Desarrollo de tecnología } \\
\hline & \multicolumn{5}{|c|}{ Adquisisción } \\
\hline & $\begin{array}{l}\text { Logística } \\
\text { interna }\end{array}$ & Operaciones & $\begin{array}{c}\text { Logística } \\
\text { externa }\end{array}$ & $\begin{array}{c}\text { Marketing } \\
\text { y ventas }\end{array}$ & $\begin{array}{l}\text { Servicio } \\
\text { posventa }\end{array}$ \\
\hline
\end{tabular}

La cadena de valor es un sistema de actividades interdependientes que se integran gracias a los eslabones. Estos eslabones son las relaciones existentes entre la manera en que se desempeña una actividad y el costo o desempeño de otra; a su vez, contribuyen a la ventaja competitiva de dos formas: la optimización, en la cual los eslabones reflejan los intercambios entre las actividades para lograr el mismo resultado general, de tal manera que una organización debe optimizar esos eslabones para reflejar la estrategia y alcanzar su ventaja competitiva y en segundo lugar, la coordinación de actividades dentro de los procesos, que contribuye a reducir los costos y a aumentar la diferenciación. Otros autores han destacado la importancia de la cadena de valor en la industria al estudiar los elementos críticos de interacción entre sus eslabones Gereffi (1999), Giuliani et al., (2005) y cómo han contribuido a la globalización y consolidación de mercados impulsando la creación de cadenas productivas locales Bair y Dussel-Peters (2006).

En Colombia, la cadena láctea está integrada tradicionalmente por tres eslabones: la producción de leche, la transformación de lácteos y la distribución-comercialización que se acoplan entre sí, generando valor agregado al producto a lo largo de toda la cadena hasta llegar al consumidor final. No obstante, en el CONPES 3676 (DNP, 2010a), la cadena de valor define seis eslabones: proveedores de insumos, sistemas productivos, centros de acopios, plantas procesadoras, comercializadores y consumidores. 


\section{Fuentes y barreras de las organizaciones asociadas con la adopción de la innovación}

\subsection{Teoría y conceptos sobre innovación}

La evolución de las teorías sobre innovación parte del concepto propuesto por Joseph Schumpeter en 1939, que consideró la tecnología y la innovación como factores externos al sistema económico. Schumpeter, al estudiar el comportamiento de los ciclos económicos, expuso que la innovación es determinante para el aumento o la disminución de la prosperidad, de tal forma que el capitalismo evoluciona en función de mecanismos endógenos presentes en los procesos de desarrollo que, a su vez, se relacionan con el crecimiento de las tecnologías (Vence, 1995).

El estudio de la innovación es bastante amplio y se relaciona con los cambios en las estructuras organizacionales, con los procesos sociales, la creatividad, el diseño, la comercialización e incluso con los cambios sistémicos en un nivel macroeconómico (Leijten, 2007). En su acepción más actual, el Manual de OSLO define la innovación así:

Una introducción de un nuevo o significativamente mejorado producto (bien o servicio), de un proceso, de un nuevo método de comercialización o de un nuevo método organizativo, en las prácticas internas de la empresa, la organización del lugar de trabajo o las relaciones exteriores. (OCDE, 2005 p. 37).

Así mismo, el Manual de OSLO presenta algunas clases de innovación según su naturaleza y enfoque, a saber:

Tabla 1. Tipos de innovación

\begin{tabular}{|l|}
\hline $\begin{array}{l}\text { Innovación en } \\
\text { producto }\end{array}$ \\
\hline $\begin{array}{l}\text { Innovación en } \\
\text { proceso }\end{array}$ \\
\hline $\begin{array}{l}\text { Innovación en } \\
\text { marketing }\end{array}$ \\
\hline $\begin{array}{l}\text { Innovación en la } \\
\text { organización }\end{array}$ \\
\hline
\end{tabular}

Es todo bien o servicio nuevo o significativamente mejorado en cuanto a sus características y funcionalidades. Estas mejoras se logran con nuevos conocimientos, tecnologías y mejoras de los materiales componentes y mediante la integración de herramientas informáticas.

Este concepto se aplica a los sectores de producción y de distribución. Son los cambios en las técnicas y en los materiales, que ayudan a mejorar la calidad y a disminuir los costes unitarios de producción o distribución.

Es el resultado de aplicar un nuevo método de comercialización que se traduzca en cambios significativos de las variables del marketing y que contribuya al éxito comercial de un nuevo producto o servicio.

Son aquellos cambios ocurridos en la dirección de las organizaciones, que influyen en su actividad, permiten un mayor acceso al conocimiento y un mejor aprovechamiento de los recursos materiales y financieros. 
Algunos autores han planteado otros tipos de innovación -como es el caso de Nelson y Winter (1982); Ettlie, Bridges y O’Keefe (1984); y Tushman y Anderson (1986)- y han estudiado la innovación incremental representada en cambios pequeños, cuyo objetivo es aumentar las prestaciones y la funcionalidad de las empresas. Estas innovaciones son poco significativas si se consideran individualmente, pero cuando se presentan de forma continua y acumulativa se convierten en una base sólida de crecimiento y progreso para las empresas. Otros autores - como Pelz (1983), Ettlie et al. (1984), Damanpour y Gopalakrishnan (2001) y Gatignon, Tushman, Smith y Anderson (2002)- hablan sobre la innovación radical, que es básicamente la ruptura de lo previamente establecido; en sí, son innovaciones de las cuales surgen nuevos productos o procesos, fuera de la evolución natural de los ya existentes, y en algunos casos son el resultado de la utilización de un principio científico nuevo que rompe con el esquema de las tecnologías anteriores.

Existe una estrecha relación entre la innovación y la tecnología. La tecnología es el medio gracias al cual el conocimiento científico se traslada a la solución de problemas concretos de una manera efectiva, por ello existe una tendencia a valorar la ciencia en términos de lo que puede aportar para la sociedad, cuyo resultado se traduce en entidades tecnológicas, es decir, en aparatos, procedimientos y habilidades (Van Wyk, 2004). Algunos autores - como Dosi et al. (1992), Child (1974), Duncan (1972), Edquist (1997), Porter (1990), Rosenberg (1994), Silverblatt y Korgaonkar (1987)- han escrito sobre la importancia de la relación entre innovación y la tecnología como fuente generadora de ventajas competitivas indispensable para la supervivencia en las organizaciones.

Tanto la adopción como la difusión son términos relacionados con la innovación y el cambio tecnológico. La adopción es un proceso mental por el que todo individuo debe pasar desde el momento en que conoce por primera vez una innovación hasta que toma la decisión final de adoptarla (Rogers, 2003). Para Rosenberg (1994), las innovaciones tecnológicas son aquellas actividades o procesos que incorporan nuevas posibilidades o alternativas técnicas en la producción, orientadas por la existencia o identificación de oportunidades de mercado o por las necesidades de la población. El origen de las innovaciones tecnológicas puede ser resultado de una invención o de la transferencia de tecnologías.

El proceso de adopción consta de una serie de etapas, elecciones y acciones que el decisor tendrá en cuenta para evaluar si la adopción de una innovación puede encajar dentro de sus prácticas habituales y para identificar qué actitudes o tareas debe desarrollar al adoptarla como son el fortalecimiento de sus capacidades internas y la vigilancia de su entorno y así disminuir esa incertidumbre (Rogers, 2003). Para Rogers (2003), la difusión se define como un proceso en el cual una innovación o tecnología se transmite a lo largo del tiempo entre los miembros de un sistema social por medio de ciertos canales de comunicación. La difusión se ve afectada por factores como el tipo de innovación (productos, procesos y tecnologías), los canales que se utilizan para comunicarla 
(interpersonales y de masas), el sistema social implicado, el tiempo y la velocidad de adopción (Rogers, 2003; Van Den Bulte, 2000). Estos factores pueden generar cambios en el comportamiento de los individuos y llevarlos a aceptar o a rechazar una innovación (Mahajan, Muller y Bass, 1990).

\subsection{Fuentes de innovación}

La innovación se relaciona con la capacidad y propensión que una persona u organización posee hacia el emprendimiento. Según Drucker (2008, p. 3), existen una serie de fuentes tanto internas como externas sobre las cuales la organización puede identificar y explotar sus potencialidades innovadoras. La tabla 2 resume los factores que Drucker identifica como fuentes generadoras de innovación tanto internas como externas para las organizaciones.

Tabla 2. Fuentes de innovación

\begin{tabular}{|l|l|}
\hline \multicolumn{1}{|c|}{ Fuentes internas } & Fuentes externas \\
\hline Eventos inesperados basados en posibles éxitos o fracasos & Demografía \\
\hline Incongruencias provenientes de lo que se espera y lo que se obtiene & Cambios en la percepción \\
\hline Necesidad de proceso y requisitos para que sean viables & Nuevo conocimiento \\
\hline Cambios en la estructura del sector o en la estructura del mercado & \\
\hline
\end{tabular}

Fuente: elaboración propia a partir de Drucker (2008).

Según Drucker (2008), dentro de los factores internos que son fuentes de innovación se encuentran eventos inesperados, incongruencias provenientes de lo que se espera y lo que se obtiene entre la economía, la industria, las preferencias y expectativas de los clientes, esto contribuye a la incertidumbre de la organización y el mercado. Otros factores internos responden a las necesidades de nuevos procesos relacionados con el desarrollo de innovaciones que serán viables cuando la organización pueda identificar una necesidad y disponga del conocimiento necesario y de la capacidad de trabajo para realizarla. En este proceso es fundamental que la organización analice su propia estructura, tenga objetivos claros, una conciencia de mejora continua y que además reconozca sus propias limitaciones. Por último, una organización puede aprovechar los cambios constantes en la estructura de la industria y el mercado con un crecimiento rápido, realizando convergencia tecnológica y creando nuevos negocios.

Los factores externos o del entorno considerados como fuentes de innovación están presentes en los cambios demográficos que deben ser aprovechados por la organización por medio de la segmentación de mercados por clientes, edades, nivel de ingresos etc. Una 
organización también puede identificar fuentes de innovación externas en la percepción de los clientes y en la aparición de nuevos conocimientos y tecnologías.

\subsection{Barreras a la innovación}

Como ya se ha mencionado, la innovación es una fuente importante para el desarrollo de ventajas competitivas en las organizaciones (Porter, 1990). Sin embargo, las organizaciones presentan una serie de barreras que limitan su capacidad para absorber conocimiento y tecnología del entorno. Algunas de las barreras a la innovación más comunes encontradas en la revisión de la literatura se exponen en la tabla 3.

Tabla 3. Barreras a la innovación

\section{La cultura innovadora}

Percepción del riesgo a invertir en $\mathrm{I}+\mathrm{D}+\mathrm{i}$

Capacidad de absorción de conocimiento y tecnología

Barreras de tipo financiero

Fuente: elaboración propia a partir de la revisión de la literatura.

\subsection{La cultura innovadora}

La I+D+i no está entre las prioridades de las PYMES, por esta razón, existe un número importante que no se involucra en proyectos de I+D (Aström, Eriksson y Arnold, 2008; Olazaran, Albizu y Otero, 2009; Roessl, Kesler y Fink, 2010). Las organizaciones no suelen acudir a los centros de innovación y desarrollo tecnológico. Esto ocurre comúnmente en PYMES de sectores con una intensidad tecnológica baja (Santamaría, García y Rialp, 2002). Además, existen otras dificultades, como la falta de confianza de las organizaciones que protegen sus conocimientos o que temen compartir su know-how interno Olazaran et al. (2009).

\subsection{Percepción del riesgo en invertir en $\mathrm{I}+\mathrm{D}+\mathrm{i}$}

Para las organizaciones, la inversión en innovación es una iniciativa especialmente riesgosa, en parte porque muchas de las innovaciones de productos no llegan al mercado y porque para desarrollarlas se requiere de un mínimo de recursos y del desarrollo de capacidades internas básicas que implican necesariamente inversiones considerables para estas organizaciones (Aström et al. 2008; Lyne, 2007; Mas-Verdú, Baviera, Puig y Martínez-Gómez, 2008; Roessl et al. 2010). La disponibilidad de las organizaciones para invertir en generación de conocimientos estará relacionada con la escala de explotación comercial y con las probabilidades de riesgo (Olazaran et al. 2009). 


\subsection{Capacidad de absorción de conocimiento y tecnología}

La capacidad de absorción es la habilidad para identificar, asimilar y explotar conocimiento del entorno y conocimiento intermedio, que es el resultado de la investigación básica, la cual proporciona una base para su posterior aplicación (Cohen y Levinthal, 1990). Por medio de la combinación de un conjunto de rutinas y procesos internos, las organizaciones pueden explotar, asimilar el conocimiento y desarrollar su capacidad de absorción (Zahra y George, 2002).

\subsection{Barreras de tipo financiero}

La incertidumbre y los altos costos asociados con el proceso innovador representan barreras para las organizaciones cuando quieren invertir en I+D+i (Silva y Ramírez, 2006). Todo proceso de cambio organizacional conlleva la incertidumbre (Moñux, Aleixandre, Gómez y Miguel, 2003), por tanto, el costo que implica investigar, que es alto para las organizaciones, no siempre puede ser asumido por ellas. Es indispensable entonces que el riesgo y el costo de la investigación sean compartidos, lo cual es posible cuando se realizan proyectos entre empresas y universidades con centros de investigación o cuando se recurre a la consecución de fondos para la investigación de carácter competitivo o en licitaciones (Aström et al. 2008),

\section{Metodología}

Esta investigación se fundamenta en un estudio descriptivo, sustentado en la revisión de la literatura existente sobre innovación, para relacionarla en el contexto rural a fin de conceptualizar y destacar los aspectos de mayor relevancia que ayuden a identificar las fuentes y barreras asociadas con la innovación en las unidades productivas de la cadena láctea.

El análisis está soportado en fuentes secundarias basadas en la revisión bibliográfica conceptual de la innovación como un paso previo al posterior levantamiento de información primaria que se realizará en la segunda fase del proyecto titulado "Innovación y energía en las unidades productivas de la cadena láctea”. Dicha fase está enmarcada en un estudio exploratorio por medio de instrumentos de medición que permitan contrastar los rasgos de competitividad en la cadena láctea.

\section{Análisis y discusión}

Las condiciones actuales de análisis de la cadena láctea evidencian una dispersión y ausencia de mecanismos de captura y análisis de información homogénea de los precios, 
costos de producción, rendimientos, consumos, informalidad, calidad de los bienes y servicios, entre otros, y representan obstáculos en cuanto a la definición de políticas de competitividad, las cuales se relacionan con niveles bajos en la configuración de valor dentro de la misma cadena productiva. En consecuencia, el desarrollo de conglomerados productivos depende de la identificación de barreras y fuentes de innovación en los diferentes eslabones de la cadena láctea para minimizar los potenciales impactos negativos y maximizar las oportunidades que los mercados internos e internacionales ofrecen. Así lo evidencia el CONPES 3675 (DPN, 2010b), en el que se afirma la importancia de gestionar "los costos de producción a través del fomento de alternativas alimenticias, el mejoramiento genético y la investigación e innovación tecnológica, [que] tienen como efecto la mejora de la productividad en el eslabón primario de la cadena” (p. 9).

Según el Programa de Trasformación Productiva (2011), Colombia ha adoptado avances tecnológicos internacionales con el propósito de mejorar en áreas como la genética, los programas de nutrición y alimentación, que han contribuido al crecimiento de la producción durante los últimos años; es decir, se identifican como fuentes innovadoras los procesos de cambio resultado de la exigencia del mercado internacional. Adicionalmente, la industria láctea colombiana ha desarrollado innovaciones en la oferta de productos, comparables con las de cualquier país desarrollado, en especial las empresas que forman parte del eslabón de comercialización, cuyas innovaciones se ven reflejadas en mejoras de empaque y presentación, modernización de los puntos de venta, que han incrementado su margen de comercialización frente a los eslabones anteriores de la cadena, así como su poder de negociación.

Así mismo, los mercados partícipes en la cadena láctea configuran prácticas con características como alto costo de producción y baja productividad de la leche, así como el alto nivel de informalidad en la comercialización, la baja capacidad de pulverización, el bajo consumo y la poca diversificación del mercado y sus efectos de integración vertical y horizontal se transforman en fuentes de innovación que permiten superar las incongruencias entre lo que se espera y lo que se obtiene en la relación de los sistemas productivos, industria de leche y expectativas de modernización, especialización y competitividad de posibles ecosistemas innovadores.

A pesar de estos esfuerzos realizados en materia de innovación en el sector lácteo colombiano, en los diagnósticos institucionales se reconoce como barrera la baja capacidad para facilitar la absorción del conocimiento y tecnología que provienen del entorno, principalmente en el eslabón primario, por ausencia tanto de tecnología como de modelos organizacionales.

En el sector lácteo, al igual que en otras industrias colombianas, hace falta un reconocimiento y comprensión por los distintos agentes de innovación (unidades productivas, Estado, centros de investigación y universidades) y de los factores con mayor influencia 
que impulsan la innovación de la cadena; los alcances del CONPES 3675 y del CONPES 3676 contribuyen inicialmente a la promoción dentro de los diferentes eslabones de una cultura innovadora y contrarrestan el imaginario pesimista de las empresas al riesgo implícito en la inversión de $\mathrm{I}+\mathrm{D}+\mathrm{i}$.

La globalización ha actuado como un factor para ayudar a hegemonizar los modos de producción y consumo en la cadena láctea, en términos de múltiples procesos, como en los aspectos económico, político y cultural, en los cuales los participantes actúan en tres niveles distintos: la multinacional, el nacional y lo local-regional; este esquema configura la creación de nuevos mercados en los que se pretende impulsar procesos de regionalización en los eslabones para especializar las actividades económicas en función de los recursos presentes en el territorio donde generen valor, por medio de una integración entre los diferentes eslabones, con mayores ventajas en economías de escala. Esta integración es el efecto de tener que cumplir con las condiciones de la competencia provocadas por un mercado global. En conclusión, se puede decir que el capital internacional fluye fácilmente a los países menos desarrollados en la inversión para la transformación industrial, la comercialización y distribución, así como para la venta de maquinaria e insumos destinados a la agricultura moderna, en un marco de sostenibilidad en el que se podría fortalecer la baja capacidad de financiación del contexto socioeconómico de la cadena láctea.

Respecto a la demografía como una fuente de innovación, en el contexto colombiano de ruralidad, las migraciones poblacionales hacia las ciudades se convierten igualmente en una debilidad del sector, al no disponer del recurso humano y joven necesario para los diferentes procesos físicos presentes en la producción de leche.

\section{Conclusiones}

De la revisión de la literatura relacionada con esta problemática, se han identificado como fuentes internas de innovación los cambios constantes en la industria y el mercado, algunos sucesos inesperados y las necesidades de nuevos procesos; y como eventos externos están los cambios demográficos, la percepción de los clientes y la aparición de nuevos conocimientos y tecnologías que a su vez promueven el cambio del entorno y contribuyen a la incertidumbre en la organización. El mismo ejercicio de revisión de la literatura permitió identificar las principales barreras asociadas con la innovación, como son la cultura innovadora, la percepción del riesgo a invertir en $\mathrm{I}+\mathrm{D}+\mathrm{i}$ y la capacidad de absorción tecnológica de las organizaciones.

En este escenario, la conformación de ecosistemas de innovación constituye una alternativa para generar transferencias de conocimiento y emprendimiento de alto impacto en 
el sector agropecuario. Lo ecosistemas de innovación se caracterizan por la interacción entre los distintos agentes que contribuyen al proceso innovador, no obstante, el riesgo es permanente si no se identifican o generan las condiciones institucionales necesarias que incentiven prácticas que dinamicen las relaciones entre dichos agentes para facilitar el desarrollo tecnológico y la innovación. Cada uno de los agentes debe cumplir una función específica dentro del proceso de innovación como sistema. El gobierno debe encargarse de establecer una política marco científica y tecnológica que garantice eficiencia en el desempeño de instituciones; en el caso de Colombia, Colciencias empodera los procesos de investigación e innovación y reserva recursos para $\mathrm{I}+\mathrm{D}+\mathrm{i}$, cuyo canal es el sector financiero. Las universidades y los centros de investigación deben proveer de los recursos materiales (laboratorios, equipos, instalaciones, etc.) al igual que del capital humano idóneo, conformado por investigadores, docentes y estudiantes.

Así mismo, el objetivo del sistema está en el rol de las empresas presentes en los distintos eslabones de la cadena láctea, de esta manera, deben participar activamente formulando proyectos de emprendimiento e innovación con universidades y centros de investigación a nivel regional, nacional e internacional, en los que propendan hacia la integración de información y conocimiento con otras empresas de diferentes sectores a fin de conformar conglomerados productivos.

\section{Referencias}

Aström, T., Eriksson, M.L. y Arnold, E. (2008). International comparison of five institute systems. Copenhagen: Forsknings-og Innovations Styrelsen. Recuperado de goo.gl/ouczdb

Azqueta, D. (2002). Introducción a la economía ambiental. Madrid: Editorial McGraw - Hill.

Bair, J., y Dussel, E. (2006). Global commodity chains and endogenous growth: Export dynamism and development in México and Honduras. World Development, 34(2), 203221. DOI: 10.1016/j.worlddev.2005.09.004

Barge-Gil, A., Santamaría, L. y Modrego-Rico, A. (2011). Complementarities between universities and technology institutes: New empirical lessons and perspectives. European Planning Studies, 19(2), 195-215. Recuperado de goo.gl/a68tW2

Becerra, M. (2012). El estado del medio ambiente de Colombia y el sector productivo. Retos y oportunidades. En F. Azuelo, A. C. González y M. L. Gutiérrez (Eds.), Tendencias en la administración. Gerencia y academia (vol. I.) Bogotá: Edición Uniandes. 
ISSN: 0124-3551 / Año 18, No 28 / enero-junio / pp. 91-110

Cárdenas, J. (2007). El desafio de las energías alternativas. En C. J. Pineda (Coord.), Futuro de la electricidad, hidrocarburos y energías alternativas. Bogotá: Editorial Politécnico Gran Colombiano.

Chesbrough, H. W. (2003). Open innovation: The new imperative for creating and profiting from technology. Boston, Massachusetts: Harvard Business School Press. Recuperado de goo. $\mathrm{gl} / \mathrm{E} 3 \mathrm{~b} 0 \mathrm{yT}$

Child, J. (1974). What determines organization. The universals vs. The it-all-depends. Organizational Dynamics, 3(1), 2-18. DOI: 10.1016/0090-2616(74)90001-1

Cohen, W. M. y Levinthal, D. A. (1990). Absorptive capacity: A new perspective on learning and innovation. Administrative Science Quarterly, 35(1), 128-152. DOI: $10.2307 / 2393553$

Consejo Nacional Lácteo. (2011). Programa de transformación productiva. Propuesta de valor cadena láctea colombiana. Bogotá: CNL. Recuperado de goo.gl/BGSLOJ

Damanpour, F. y Gopalakrishnan, S. (2001). The dynamics of the adoption of product and process innovations in organizations. Fournal of Management Studies, 38(1), 45-65. DOI: $10.1111 / 1467-6486.00227$

Departamento Nacional de Planeación (DNP) (2011). Plan Nacional de Desarrollo 20102014. Prosperidad para Todos. Bogotá: DNP.

- (2010a). Documento CONPES 3676. Consolidación de la política sanitaria y de inocuidad para las cadenas láctea y cárnica. Bogotá: DNP. Recuperado de goo.gl/y7uP5o . (2010b). Documento CONPES 3675. Politica nacional para mejorar la competitividad del sector lácteo colombiano. Bogotá: DNP. - (2009). Documento CONPES 3582. Política Nacional de Ciencia, Tecnología e Innovación. Bogotá: DNP.

Dosi, G., Giannetti, R. and Toninelli, P. (1992). Technology and Enterprise in a Historical Perspective. Clarendon Press, Oxford.

Duncan, R. (1972). Characteristics of organizational environments and perceived environmental uncertainty. Administrative Science Quarterly, 17(3), 313-327. DOI: $10.2307 / 2392145$

Drucker, P. (2008). Management. Revised Edition. Zondervan. 
Edquist, C. (1997). Systems of innovation. Technologies, institutions and organizations. Londres: Pinter/Cassell.

Ettlie, E., Bridges, W. y O’Keefe, R. (1984). Organizational strategy and structural differences for radical vs. incremental innovation. Management Science, 30(6), 682-695.

Foro Económico Mundial. (2016). Competitiveness Rankings. Recuperado de goo.gl/gNjBj2

Gatignon, H., Tushman, M. L., Smith, W. y Anderson, P. (2002). A structural approach to assessing innovation: Construct development of innovation locus, type and characteristics. Management Science, 48(9), 1103-1122.

Gaviria, D. (2012). Cambio climático: retos y oportunidades para las empresas latinoamericanas. En F. Azuelo, A. C. González y M. L. Gutiérrez (Eds.), Tendencias en la administración. Gerencia y academia (vol. I). Bogotá: Ediciones Uniandes.

Gereffi, G. (1999). International trade and industrial upgrading in the apparel commodity chain. Journal of International Economics, 48(1), 37-70. DOI: 10.1016/ S0022-1996(98)00075-0

Giuliani, E., Pietrobelli y Rabellotti, R. (2005). Upgrading in global value chains: Lessons from Latin American clusters. World Development, 33(4), 549-573. DOI:10.1016/j. worlddev.2005.01.002

Hirschman, A. (1958). The strategy of economic growth. Yale New Haven.

Hobday, M. (2005). Firm-level innovation models: Perspectives on research in developed and developing countries. Technology Analysis E Strategic Management, 17(2), 121-146. DOI: $10.1080 / 09537320500088666$

Intergovernmental Panel on Climate Change (IPCG). (2007). Cambio climático 2007. Informe de sintesis. Contribución de los grupos de trabajo I, II, II al Cuarto Informe de Evaluación del Grupo Intergubernamental de Expertos sobre Cambio Climático. Ginebra, Suiza: IPCC.

Kaplinsky, R y Morris, M. (2000). A Handbook for value chain research. Ottawa: International Development Research Center.

Krugman, P. (1999). Balance sheets, the transfer problem, and financial crises. In International finance and financial crises (pp. 31-55). Springer Netherlands.

Krugman, P., Obstfield, M., Melitz, M. (2012). Economia internacional: Teoría y política. Editorial Pearson. 
Leijten, J. (2007). The future of RTOs: A few likely scenarios. En European Comisión, The future of key research actors in the European research área (pp. 119-138). Bélgica: EU.

Lyne, MB. (2007). Research institutes have become industry partners: These institutes have evolved into competitive players in the market for technical solutions to industrial problems. Research Technology Management, 50(4), 42-48.

Mahajan, V., Muller, E. y Bass, F. (1990). New products diffusion model in marketing: A review and directions for research. Fournal of Marketing 54(1), 1-26. DOI: $10.2307 / 1252170$

Mas-Verdú, F., Baviera-Puig, A. y Martínez-Gómez, V. (2008). Internacionalización, servicios y política de innovación: el papel de los centros tecnológicos. Información Comercial Española, ICE: Revista de Economía, 844, 155-165. Recuperado de goo. $\mathrm{gl} / 42 \mathrm{~T} 4 \mathrm{On}$

Menke. M., Xu, Q. y Gu, L. (2007). An analysis of the universality, flexibility, and agility of total innovation management: A case study of Hewlett-Packard. Fournal of Technology Transfer, 32(1), 49-62. DOI: 10.1007/s10961-006-9005-z

Ministerio de Ambiente, Vivienda y Desarrollo Territorial. (2010). Política Nacional para la Gestión Integral del Recurso Hídrico. Bogotá: Autor.

Molina-Morales, X. y Mas-Verdú, F. (2008). Intended ties local institutions as factors in innovation: An application to Spanish manufacturing firms. European Planning Studies, 16(6), 811-827. DOI: 10.1080/09654310802079452

Moñux, D., Miguel, L., Aleixandre, G. y Gómez, F. (2003). Evaluación del impacto social de proyectos de $I+D+i$. Guía práctica para centros tecnológicos. Valladolid: CARTIF y Departamento de Ingeniería de Sistemas y Automática (Universidad de Valladolid). Recuperado de goo.gl/WgOhU8

Nelson, R. y Winter, S. (1982). An evolutionary theory of economic change. Cambridge: MA: Harvard University Press.

Nobelius, D. (2004). Towards the sixth generation of R\&D management. International Journal of Project Management, 25(5), 369-375. DOI: 10.1016/j.ijproman.2003.10.002

OCDE. (2005). Manual de OSLO. Guía para la recogida e interpretación de datos sobre innovación (3.a ed.) (Trad. Grupo Tragsa). España: OCDE.

Olazaran, M., Albizu, E. y Otero, B. (2009). Technology transfer between technology centres and SMEs: Evidence from the Basque County. European Planning Studies, 17(3), 345-363. DOI: 10.1080/09654310802618010 
Pelz, D. (1983). Quantitative case histories of urban innovation: Are there innovating stages? IEEE Transactions on Engineering Management, 30(2), 60-67. DOI: 10.1109/ TEM.1983.6447503

PNUD Colombia. (2011). Colombia rural. Razones para la esperanza (Informe Nacional de Desarrollo Humano 2011). Bogotá: INDH - PNUD. Recuperado de goo.gl/vOM6fj

Porter, M. (2002). La ventaja competitiva de las naciones: creación y sostenimiento de un desempeño superior (2.a ed.). México: Editorial CECSA.

. (1990). The competitive advantage of nations. Nueva York: The Free Press.

. (1985). The competitive advantage: Creating and sustaining superior performance. Nueva York: The Free Press.

Roessl, D., Kessler, A. y Fink, M. (2010). The role or research and technology organizations in innovation processes or small and medium-sized enterprises. The International Journal of Entrepreneurship and Innovation, 11(3), 199-207. DOI: $10.5367 / 000000010792217290$

Rogers, E. (2003). Diffusion of innovations (5.a ed.). Nueva York: The Free Press.

Rosas-Baños, M. (2013). Nueva ruralidad desde dos visiones de progreso rural y sustentabilidad: economía ambiental y economía ecológica. Polis. Revista Latinoamericana, 12(34), 225-241. DOI: 10.4067/S0718-6568201300010001

Rosenberg, N. (1994). Incertidumbre y cambio tecnológico. Revista de Historia Industrial, 6, 11-30. Recuperado de goo.gl/zeAjje

Rothwell, R. (1994). Towards the fifth-generation innovation process. International Marketing Review, 11(1), 7-31. DOI: 10.1108/02651339410057491

Santamaría, L., García, M. y Rialp, J. (2002). Caracterización de las empresas que colaboran con centros tecnológicos (Documentos de trabajo n.o 2005/5, UAB). Barcelona: Universidad Autónoma de Barcelona. Recuperado de goo.gl/BcMj0S

Silva, G. y Ramírez, A. (2006). Análisis de eficiencia de institutos tecnológicos de España y Brasil: una aplicación del análisis envolvente de datos (DEA). Fournal of Technology Management $\mathfrak{E}$ Innovation, 1(4), 43-56. Recuperado de goo.gl/QgYIvb

Silverblatt, R. y Korgaonkar, P. (1987). Strategic market planning in a turbulent business environment. Fournal of Business Research, 15(1), 339-358. DOI: 10.1016/0148-2963(87)90005-1 
ISSN: 0124-3551 / Año 18, No 28 / enero-junio / pp. 91-110

Stern, N. (2007). El informe Stern. La verdad del cambio climático. Barcelona: Ediciones Paidós Ibérica, S. A.

Tushman, M. y Anderson, P. (1986). Technological discontinuities and organizational environments. Administrative Science Quarterly, 31(3), 439-465. DOI: 10.2307/2392832

Van Den Bulte, C. (2000). New product diffusion acceleration: Measurement and analysis. Marketing Science, 19, 366-380. DOI: 10.1287/mksc.19.4.366.11795

Van Wyk, R. (2004). Technology, a unifying code: A simple and coherent view of technology. Cape Town: Stage Media Group.

Vence Deza, J. (1995). Economía de la innovación y del cambio Tecnológico. Editorial siglo XXI. España.

Zahra, S. A. y George, G. (2002). Absorptive capacity: A review, reconceptualization, and extension. Academy of Management Review, 27(2), 185-203. 